\title{
Inteligencia artificial: un punto de encuentro para todos
}

\author{
Guadalupe Vadillo Bueno
}

"La IA está en todas partes.

No es esa cuestión temible

y enorme en el futuro.

La ia está aquí con nosotros".

Fei-Fei Li

Este número no sólo es una colección de artículos: se trata de una llamada que nos convoca a pensar cómo estamos constantemente en contacto con agentes que usan inteligencia artificial (iA) y cómo su presencia e impacto en nuestra vida serán cada vez más amplios. Miles de artículos y noticias sobre este tema se publican a diario, sin embargo, es probable que no tengamos claridad sobre su estado de desarrollo, sus posibilidades de afectar nuestro contexto, sus retos y amenazas... Y es que hasta definirla resulta complejo, ya que existen enfoques variados y posturas contrapuestas. Se ha señalado incluso un efecto IA que consiste en que cuando sus desarrollos llegan al público general y se "normalizan" dejan de considerarse inteligencia como tal (Haenlein y Kaplan, 2019). Este efecto hace difícil contar con definiciones de IA que sean aceptadas universalmente. A esto se añade la complejidad de sus estados de evolución: desde la IA estrecha, pasando por la general, hasta la superinteligencia, así como de las competencias cognitivas, emocionales y sociales que integra (analítica, inspirada en humanos o humanizada) (Kaplan y Haenlein, 2020).

Dol: http://doi.org/10.22201/codeic.16076079e.2020.v21n1.a0 
En general, se reconoce la importancia de saber de IA, de explorar sus posibilidades en el ámbito de cada persona o entidad y de ponerla en acción por ejemplo, Liang, Lee y Workman, 2020, en el campo de la moda; Vercauteren, Unberath, Padoy y Navab, 2020 en medicina o Smith, 2020, en agricultura, por citar solo algunas áreas en que la ı tiene un impacto-. Hay quienes ya no sólo se quedan en ese nivel, como Lichtenhaler (2020), quien indica que las organizaciones deben considerar la inteligencia humana (IH), la artificial y la metainteligencia. Esta última renueva y recombina IA y ıн para lograr transformaciones, dentro de la organización, de carácter incremental, modular, en su arquitectura de inteligencia y, en el más alto nivel, de tipo radical.

Le proponemos a nuestros lectores un banquete de enfoques y cuestionamientos sobre la IA. Degustarlo implicará reflexiones sobre las posibilidades inmediatas y de largo plazo que nos plantea, las dimensiones éticas que necesariamente se derivan de su introducción en nuestras vidas, y sobre cómo cambiaremos al tenerla cada vez más cerca en todas las esferas de nuestro acontecer diario.

En el área de humanidades, empezamos con el análisis que el especialista en decodificación de la imagen Alfonso Miranda hace sobre la IA en el arte. En el campo de la literatura, este número de la Revista Digital Universitaria (RDU) cuenta con un relato transmedia de una maravillosa escritora, la Dra. María Luisa Zorrilla, que explora las posibilidades de la iA en el área de la creación. El número se enriquece con un artículo desarrollado por la Dra. Jackie Bucio donde postula que la inteligencia colectiva derivada de una experiencia de crowdsourcing, unida a una IA, puede crear supermentes que aportan mayor beneficio que sólo un tipo de inteligencia. Así, describe una experiencia de la escenógrafa experimentadora Es Devlin en Londres. En su instalación, los paseantes aportan palabras para que un quinto león en la Plaza de Trafalgar presente poemas generados por una red neuronal que aprendió a escribir después de leer 25 millones de palabras de poesía del siglo xIx.

Imagen 1. Contenidos del número 1, volumen 21 de la RDU. Imagen elaborada a partir de una foto de Pixabay.

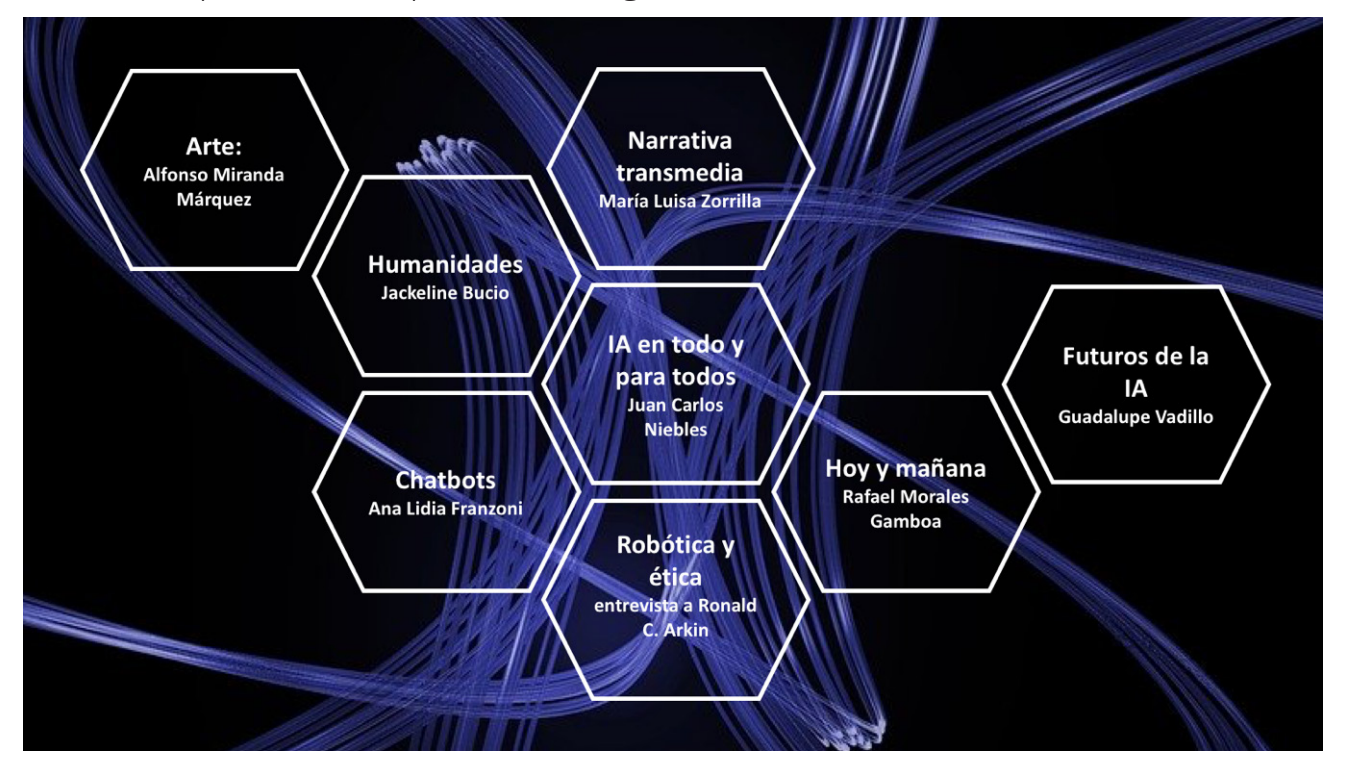


La Dra. Ana Lidia Franzoni, a partir de una entrevista que hizo con Mitsuku, un chatbot que dice ser una chica inglesa de 18 años, nos introduce en los tipos de respuestas que tienen dichas tecnologías conversacionales, cómo se crean y qué tan lejos estamos de poder realmente conversar con ellas.

El Dr. Juan Carlos Niebles, colombiano que trabaja para la Universidad de Stanford, sintetiza en una entrevista gran parte de lo que se postula en este número: la IA va a cambiar al mundo. Presenta el programa de Stanforfd Al4ALL, que tiene el propósito de promover una participación plural con alta representación de chicas en esta área.

Como un complemento a este número, un colectivo del Bachillerato a distancia de la unam invitó al Dr. Ron Arkin, del Georgia Institute of Technology, a una entrevista en la que compartió un poco de su amplia experiencia sobre robots y la ética que involucra su desarrollo y uso en diversas actividades humanas, incluyendo la guerra.

El Dr. Rafael Morales hace una detallada presentación de 25 visiones sobre la IA en un artículo sobre su presente y futuro. A partir del libro Possible Minds: Twenty-Five Ways of Looking at IA, describe, contrasta y organiza los planteamientos de especialistas de una variedad de disciplinas para confrontarnos ante ideas como el impacto de la ia en nuestra vida de trabajo, la pérdida de privacidad que podemos sufrir o las tres leyes de la IA.

Por último, lanzando una mirada hacia el futuro, cuestionamos a cuatro expertos mexicanos en este campo y revisamos algunos estudios sobre posibles escenarios para intentar identificar las aportaciones y riesgos que perciben en el horizonte. Algunos hablan de codependencia, otros de una IA peligrosa, capaz de provocar riesgos catastróficos. No obstante, prácticamente todos identifican muchos beneficios en muy diversos ámbitos de la actividad humana.

La experiencia de lectura de estas colaboraciones será única y tendrá resonancias distintas en cada lector, de acuerdo con sus posturas y expectativas con relación a la IA. Lo que seguramente será común es el enriquecimiento y el interés por conocer más de este tema, y la conclusión de que más nos vale saber de inteligencia artificial.

\section{Referencias}

* Haenlein, M. y Kaplan, A. (2019). A brief history of artificial intelligence: on the past, present and future of artificial intelligence. California Management Review, 61(4), 5-14. Dol: https://doi.org/10.1177/0008125619864925.

* Kaplan, A. y Haenlein, M. (2020). Rulers of the world, unite! The challenges and opportunities of artificial intelligence. Business Horizons, 63(1), 37-50. Dol: https:// doi.org/10.1016/j.bushor.2019.09.003. 
* Liang, Y., Lee, S. H. y Workman, J. E. (2020). Implementation of artificial intelligence in fashion: Are consumers ready? Clothing and Textiles Research Journal, 38(1), 3-18. Dol: https://doi.org/10.1177/0887302X19873437.

* Lichtenhaler, U. (2020). Beyond artificial intelligence: Why companies need to go the extra step. Journal of Business Strategy, 41(1), 19-26. Dol: https://doi.org/10.1108/ IBS-05-2018-0086.

* Smith, M. J. (2020). Getting value from artificial intelligence in agriculture. Animal Production Science, 60, 46-54. Dol: https://doi.org/10.1071/AN18522.

* Vercauteren, T., Unberath, M., Padoy, N. y Navab, N. (2020). CAI4CAl: The Rise of Contextual Artificial Intelligence in Computer-Assisted Interventions. Proceedings of the IEEE, 106(1), 198-214. Dol: https://doi.org/10.1109/JPROC.2019.2946993.

\section{Cómo citar este artículo}

* Vadillo Bueno, Guadalupe (2020). Inteligencia artificial: un punto de encuentro para todos. Revista Digital Universitaria (RDU). Vol. 21, núm. 1 enero-febrero. Dol: http://doi.org/10.22201/codeic.16076079e.2020.v21n1.a0. 